\title{
A Neutral Hydrogen Survey of the Large Magellanic Cloud: Aperture Synthesis and Multibeam Data Combined
}

\author{
Sungeun Kim ${ }^{1}$, Lister Staveley-Smith ${ }^{2}$, Michael A. Dopita ${ }^{3}$, Robert J. Sault ${ }^{2}$, \\ Kenneth C. Freeman ${ }^{3}$, Youngung Lee ${ }^{4}$, and You-Hua $\mathrm{Chu}^{5}$
}

\begin{abstract}
Recent H I surveys of the Large Magellanic Cloud (LMC) with the Australia Telescope Compact Array (Kim et al. 1998) and the Parkes multibeam receiver (Staveley-Smith et al. 2003) have focussed, respectively, on the small-scale $\left(<20^{\prime}\right)$ structure of the interstellar medium (ISM) and the large-scale $\left(>1^{\circ}\right)$ structure of the galaxy. Using a Fourier-plane technique, we have merged both data sets providing an accurate set of images of the LMC sensitive to structure on scales of $15 \mathrm{pc}$ (for an LMC distance of $50 \mathrm{kpc}$ ) upwards. The spatial dynamic range (2.8 orders of magnitude), velocity resolution $\left(1.649 \mathrm{~km} \mathrm{~s}^{-1}\right.$ ), brightness temperature sensitivity $(2.4 \mathrm{~K})$ and column density sensitivity $\left(8.9 \times 10^{18} \mathrm{~cm}^{-2}\right.$ per $1.649 \mathrm{~km} \mathrm{~s}^{-1}$ channel) allow for the studies of phenomena ranging from the galaxy-wide interaction of the LMC with its close neighbours to the small-scale injection of energy from supernovae and stellar associations into the ISM of the LMC. This paper presents the merged data and the size spectrum of H I clouds which is similar to the typical size spectrum of the holes and shells in the H I distribution.
\end{abstract}

Subject headings: galaxies: individual (Large Magellanic Cloud) — galaxies: ISM — ISM: neutral hydrogen — Magellanic Clouds — radio lines: galaxies

\footnotetext{
${ }^{1}$ University of Massachusetts, Astronomy Department, 710 N. Pleasant St., Amherst, MA 01003; email: skim@daisy.astro.umass.edu

${ }^{2}$ Australia Telescope National Facility, 76 Epping, NSW 2121, Australia; email:1stavele@atnf.csiro.au,rsault@atnf.csiro.au

${ }^{3}$ Mount Stromlo and Siding Spring Observatories, PO Box, Canberra, ACT2611, Australia; email:mad@mso.anu.edu.au; kcf@mso.anu.edu.au

${ }^{4}$ Korea Astronomy Observatory, Taeduk Radio Astronomy Observatory, Whaam-dong 61-1, Yusong-gu, Taejon, 305-348, Korea; email:yulee@trao.re.kr

${ }^{5}$ Astronomy Department, University of Illinois at Urbana-Champaign, 1002 West Green st., IL61801; email:chu@astro.uiuc.edu
} 


\section{Introduction}

High resolution $\mathrm{H}$ I observations of nearby galaxies allow the study of many aspects of their dynamics, morphology and interstellar physics. Measurement of the vertical H I distribution and velocity dispersion, for example, allows the mass-to-light ratio and dark matter content of galaxy disks to be studied (Olling 1996). The shape of the inner velocity field, using $\mathrm{H}$ I as a tracer, can discriminate between different halo models (de Blok et al. 2001). Also, the relationship between H II regions and H I holes says much about the evolution of galaxies and the propagation of star-forming regions (Walter \& Brinks 2001).

The LMC, as the nearest gas-rich galaxy to our own, has been the subject of several H I studies (Luks \& Rohlfs 1992; McGee \& Milton 1966). The advantage of studying the LMC in $\mathrm{HI}$ is that it is the nearest galaxy to our own with a distance of 50-55 kpc (Feast 1991), it is presented nearly face-on, and it is a very gas-rich and active star forming galaxy, so that it allows for a detailed study of the structure, dynamics and the interstellar medium of a star-forming galaxy at close range. The early single-dish observations revealed the existence of expanding H I shells in the LMC (Westerlund \& Mathewson 1996; McGee \& Milton 1966). A very spectacular example of such a shell associated with the major star-forming region Constellation III was described by Dopita, Mathewson, \& Ford (1985).

However, its location in the far south means that high resolution studies had to await the advent of the Australia Telescope Compact Array $^{1}$ (ATCA). The advent of ATCA finally allowed us to take full advantage of the aperture synthesis technique in southern hemisphere observations, and our recent high-resolution H i survey of the Large Magellanic Cloud (LMC) revealed that the structure of the neutral atomic interstellar gas to be dominated by numerous shells and holes as well as complex filamentary structure (Kim et al. 1998). Its huge angular scale ( $\sim 8^{\circ}$ for the inner disk), required effective observing techniques and mosaicing methods (Staveley-Smith et al. 1997), and required the development of new deconvolution algorithms (Sault, Staveley-Smith, \& Brouw 1996).

At the largest scales, understanding the interaction of the LMC with the Galaxy and the Small Magellanic Cloud (SMC) is important (Putman et al. 1998; Weinberg 2000). Also important is knowing the viewing geometry of the disk of the LMC (van der Marel \& Cioni 2001), its mass and dark matter content (Kim et al. 1998; Alves \& Nelson 2000) and the origin of the off-center bar (Zhao \& Evans 2000; Gardiner, Turfus, \& Putman 1998). At intermediate scales, the origin of the supergiant shells is tantalizing. Are supernovae

\footnotetext{
${ }^{1}$ The Australia Telescope Compact Array is funded by the is funded by the Commonwealth of Australia for operation as a National Facility managed by CSIRO.
} 
and stellar winds (Dopita, Mathewson, \& Ford 1985; Chu \& Mac Low 1990) sufficient, or are other factors such as instabilities in the ISM (Wada et al. 2000), high-velocity cloud collisions (Tenorio-Tagle \& Bodenheimer 1980), or gamma ray bursts (Efremov, Ehlerova, \& Palous 1999) important? At smaller scales, the structure of the ISM, the structure of photo-dissociation regions and the detailed feeding and feedback of star-forming regions are important for understanding their evolution.

In this paper, we perform the last step of our high-resolution H I survey of the LMC by combining the already-made ATCA observations (Kim et al. 1998) with the relatively newer observations made with the multibeam receiver (Staveley-Smith et al. 2002) on the Parkes telescope. This step is necessary in order to provide a single-dish data cube containing structure over the full range of spatial frequencies on the sky. The ATCA images, on their own, may not provide reliable H I masses for objects extended over more than $10^{\prime}-20^{\prime}$ as the minimum ATCA baseline for these observations was $30 \mathrm{~m}$, for which the fringe spacing is $\lambda / D=24^{\prime}$ (though mosaicing extends the effective fringe spacing to $\sim 36^{\prime}$ - see Holdaway 1999). Similarly, Parkes H I images have a resolution of $14^{\prime}-16^{\prime}$, depending on the details of the gridding of the data, and are therefore not useful for examining structures smaller than $\sim 25^{\prime}$. Several examples of combination of aperture synthesis and single-dish data can be found in the literature: Ye \& Turtle (1991) and Stanimirović et al. (1999) utilize imageplane combination, whereas Bajaja \& van Albada (1979), and Wilner \& Welch (1994) utilize Fourier-plane combination. In this paper, we further discuss and develop data combination techniques, and use the most suitable to merge the H I data for the LMC.

In $\S 2$ in this paper, we summarize the existing $\mathrm{H}$ I data sets from the ATCA and Parkes telescopes and describe the method for linearly combining synthesis and single-dish observations. In $\S 3$, we present the combined data set in the form of spatial-spatial map integrated over the full velocity range, velocity channel maps, and spatial-velocity maps at forty galactic longitude. In $\S 4$, we confirm the $\mathrm{H}$ I giant and supergiant shell candidates selected from the ATCA data using the new fully-sampled data, and then compare the size distribution of $\mathrm{H}$ I shells with the size distribution of discrete H I clouds in the LMC. In $\S 5$, we give a summary of this paper.

\section{Observation}

The Australia Telescope Compact Array (ATCA) has been used in mosaic mode to survey a region $10^{\circ} \times 12^{\circ}$ covering the $\mathrm{LMC}$ and at an angular resolution of 1 '.0, corresponding to a spatial resolution of $15 \mathrm{pc}$ in the LMC. The detailed observations and data reductions are described in Kim et al. (1998). The efficient observing techniques and mosaicing methods 
with the ATCA are described in Staveley-Smith et al. (1997).

The observing band was centered on $1.419 \mathrm{GHz}$, corresponding to a central heliocentric velocity coverage from -33 to $+627 \mathrm{~km} \mathrm{~s}^{-1}$, at a velocity resolution of $1.65 \mathrm{~km} \mathrm{~s}^{-1}$. Imaging of the observed visibility data has been performed using the standard linear mosaicing scheme (Sault, Staveley-Smith, and Brouw 1996). After deconvolution the final cube was constructed by convolving the maximum entropy model with a circular Gaussian beam of FWHM $1^{\prime} \times 1^{\prime}$ and adding the deconvolution residuals back in. The RMS noise of the final map, determined from the line-free parts of the final data cube, is $31 \mathrm{mJy}^{\mathrm{beam}^{-1}}$. The corresponding brightness temperature sensitivity is $2.5 \mathrm{~K}$, which is equivalent to a column density sensitivity of $1.6 \times 10^{19} \mathrm{~cm}^{-2}$ per $1.649 \mathrm{~km} \mathrm{~s}^{-1}$ channel.

\subsection{Parkes $\mathrm{H}_{\text {I }}$ Observations}

To provide the complete map at low spatial resolution to complement the ATCA data, observations were taken with the inner 7 beams of the Parkes Multibeam receiver (StaveleySmith et al. 1996) on 1998 December 13 to 17 (Staveley-Smith et al. 2001). The receiver was scanned across the LMC in orthogonal east-west and north-south great circles and the receiver was continuously rotated such that the rotation angle was always at $19^{\circ} .1$ to the scan trajectory, thus ensuring uniform spatial sampling of the sky. The area covered was $13^{\circ}$ by $14^{\circ}$ in RA and Dec., respectively, and centered on $05^{\mathrm{h}} 20^{\mathrm{m}},-68^{\circ} 44^{\prime}(\mathrm{J} 2000)$. In a single scan, the spacing between adjacent tracks is 9.5 , which is smaller than the mean FWHP beam width of 14 !' 1 , but greater than the Nyquist interval $(\lambda / 2 D)$ of 5.7 . Therefore, six scans are interleaved in each of the principal scan directions, resulting in a final track spacing of 1'.6. In total, $12 \times 6 \mathrm{RA}$ scans and $11 \times 6$ Dec. scans were made. Seven scans were dropped or edited out due to drive problems, leaving a total of 131 scans consisting of a total of 29 hours of on-source integration on each of 7 beams. The average integration time per beam area is 360 seconds for both polarizations.

The scan rate of the telescope was $1.0 \mathrm{~min}^{-1}$ and the correlator was read every 5 seconds. Therefore, the beam was slightly broadened in the scan direction to 14.5 . After averaging orthogonal scans, the effective beam width reduces to $14^{\prime} .3$. The central observing frequency was switched between 1417.5 and $1421.5 \mathrm{MHz}$, again every 5 seconds. This allowed the bandpass shape to be calibrated without spending any time off-source. A bandwidth of 8 $\mathrm{MHz}$ was used with 2048 spectral channels in each of two orthogonal linear polarizations. H I emission from the LMC appeared within the band, at both frequency settings. After bandpass calibration, the data from both settings were shifted to a common solar barycentric

reference frame. The velocity spacing of the multibeam data is $0.82 \mathrm{~km} \mathrm{~s}^{-1}$, but the final 
cube was Hanning-smoothed to a resolution of $1.6 \mathrm{~km} \mathrm{~s}^{-1}$. The useful velocity range in the final cube (i.e. after excluding frequency side lobes of the LMC and the Galaxy, and band-edge effects) is -66 to $430 \mathrm{~km} \mathrm{~s}^{-1}$.

Bandpass calibration, velocity shifting and preliminary spectral baseline fitting were all done using the AIPS $^{++}$LiveDATA task. Subsequently, the data were convolved onto a grid of $4^{\prime}$ pixels using a Gaussian kernel with a FWHP of 8'.0. This broadens the effective, scan-broadened, beam width of the inner 7 beams from 14'3 to about 16'4. Residual spectral baselines were removed by fitting polynomials in the image domain (MIRIAD task CONTSUB). The multibeam data were calibrated relative to a flux density for PKS B1934-638 of 14.9 Jy at the observing frequency. The brightness temperature conversion factor of $0.80 \mathrm{~K} \mathrm{Jy}^{-1}$ was established by an observation of $\mathrm{S} 9\left(T_{B}=85 \mathrm{~K}\right.$, Williams 1973). On the same scale, we measured a brightness temperature for pointing 416 in the SMC $\left(00^{\mathrm{h}} 47^{\mathrm{m}} 52.6^{\mathrm{s}},-73^{\circ} 02^{\prime} 19^{\prime \prime} .8\right.$, J2000) of $T_{B}=133 \mathrm{~K}$, compared with the $137 \mathrm{~K}$ measured by Stanimirović et al. (1999). The $3 \%$ difference is probably due to the different characteristics of the feeds used in the two observations, and residual uncertainties in absolute bandpass calibration. The rms noise in the line-free region of the cube is $27 \mathrm{mK}$, which is close to the theoretical value.

\subsection{Combining ATCA and Parkes Observations}

Several techniques are available to combine the interferometric and single-dish data. The data can be combined during a joint maximum entropy deconvolution operation. Alternatively the single-dish data can be used as a 'default' image in a maximum entropy deconvolution of the interferometer data. Another possibility is to feather together (a linear merging process) the single-dish and interferometer images. Stanimirovic et al. (1999) have used an approach where the interferometer and single-dish image are added together before deconvolution, and then deconvolution performed with a modified point-spread function. Tests by Stanimirovic et al. (1999) found that the different techniques produced quite similar results. Given this, and as we already had a deconvolved interferometric cube, and as the computational requirements to re-perform a deconvolution of these data is large, we have used an image feathering approach.

The technique we have used is a variant of the approach described by Schwarz \& Walker (1991). As the Parkes and ATCA images give accurate representations of the LMC at short spacings and mid to long spacings respectively, a composite image can be formed by filtering out the short spacing data from the ATCA image, and then adding the Parkes image. This process is most easily visualized in the Fourier domain as in Figure 1 shows the expected amplitude as a function of spatial frequency of a point source for our observations. The 
Fourier transform of the Parkes images were added to the final images with no weighting (i.e. 'natural' weight in interferometric nomenclature). The deconvolved ATCA data was also Fourier-transformed, but by down-weighting the lower spatial frequencies such that the combined weight of the Parkes and ATCA data was the same as the response to a $1 .^{\prime} 0$ Gaussian. The MIRIAD task IMMERGE was used.

Before combining, the Parkes image was interpolated onto the same coordinate grid as the ATCA mosaicked image. Also the residual primary beam attenuation remaining in the ATCA image was applied to the Parkes image (the mosaicing process we have used does not perform full primary beam correction when this would result in excessive noise amplification). In order to perform the combination of the Parkes and ATCA observations, we need to ensure that the flux calibration between the two data types are consistent. Ideally we would like to find the ratio of the flux density of an unresolved point source in the field. We have estimated this calibration factor by examining data in the Fourier plane between 21 and 31 meters - data in this annulus is well measured by the Parkes and mosaicked ATCA observations. After tapering the ATCA data to the same resolution as the Parkes data, we found a scale factor of 1.3 minimized the $L_{1}$ difference between the interferometer and singledish Fourier components (the real and imaginary parts of the data where treated as distinct measurements in the fitting). Note that the scale factor (and indeed the entire feathering process) requires a good estimate of the resolution of the Parkes image. The effective beam size of 16.9 arcmin was adopted as it gave a scale factor independent of spatial frequencies (Stanimirovic et al. 1999).

The resolution of the combined H I image of the LMC is $1 .^{\prime} 0$ which is the same as for the ATCA interferometer map. The RMS noise of the combined map, determined from the

line-free parts of the final data cube, is $\sim 19$ mJy beam $^{-1}$. This corresponds to a brightness temperature sensitivity of $\sim 2.4 \mathrm{~K}$.

\section{DATA PRESENTATION}

To assist the comparison, we display in Figure 2 the individual channel maps from the combined H I ATCA map with the Parkes single dish map. This figure can be compared with Figure 2 of Kim et al. (1998). The individual channel maps have a velocity resolution of $1.649 \mathrm{~km} \mathrm{~s}^{-1}$ and cover a velocity range of $V_{H E L}=205 \mathrm{~km} \mathrm{~s}^{-1}$ to $V_{H E L}=334 \mathrm{~km} \mathrm{~s}^{-1}$. However, the H I emission is detected mostly in the velocity range of $V_{H E L}=190-387 \mathrm{~km} \mathrm{~s}^{-1}$ . The peak H I surface brightness image and the column density image of the LMC are shown in Figure 3 and Figure 4. The peak brightness temperature is $136.7 \mathrm{~K}$ at RA $=05^{\mathrm{h}} 40^{\mathrm{m}} 43^{\mathrm{s}}$, $\mathrm{DEC}=-69^{\circ} 48^{\prime} 49.7^{\prime \prime}(\mathrm{J} 2000)$. The peak column density of $7.3 \pm 0.3 \times 10^{21} \mathrm{~cm}^{-2}$ in the 
$V_{H E L}=225-310 \mathrm{~km} \mathrm{~s}^{-1}$ assuming that the $\mathrm{H} \mathrm{I}$ is optically thin at this point.

There is a remarkable correspondence between the features of this map and the $\mathrm{HI}$ emission obtained from the previous ATCA survey (Kim et al. 1998). For example, the spiral structure is clearly seen in the individual channel maps (Figure $2 \mathrm{~b}$ ) and the peak H I surface brightness map (Figure 3) as well as its integrated map (Figure 4). In contrast to the optical image of the LMC (Kim et al. 1999, Figure 5a), both ATCA map and ATCA+Parkes combined map show that the $\mathrm{H}$ I distribution is uniform and no bar feature corresponding to the optical one. The huge H I hole of diameter about $1.2 \mathrm{kpc}$ resides between the southern (de Vaucouleurs \& Freeman 1972) and northern spiral arm.

The large-scale distribution of hydrogen in 30 Dor region and the south of 30 Dor region in the coordinate range $05^{h} 49^{m}<\mathrm{RA}<05^{\mathrm{h}} 36^{\mathrm{m}},-73^{\circ} 00^{\prime}<$ DEC $<-68^{\circ} 30^{\prime}$ displays two large sheets of gas having a relative difference in the line-of-sight velocity about 40 $\mathrm{km} \mathrm{s}^{-1}$ but with relatively small internal velocity dispersions. The general shape of this feature seen in the Position-Velocity $(P-V)$ diagram (Figure 5) can be explained as the disk (D-) component and a second surface (L-component), discussed by Luks and Rohlfs (1992), which is possibly a region affected by the ram-pressure associated with the motion of the LMC through the outer halo of our Galaxy (Kim et al. 1998). However, many arclike structures seen in the $P-V$ diagram are likely to originate from expanding shells (Kim et al. 1999), although it is very difficult to distinguish between the components from expanding shells and intrinsic separate disk components (e.g., the L- and D- components) as a result of combined action of multiple expanding shells and local random motion.

\section{SIZE SPECTRUM OF H I SHELLS AND CLOUDS}

The structure of the neutral atomic ISM in the LMC shows a complex distribution of $\mathrm{H}$ I emission, which is chaotic with hundreds of clouds, shells, arcs, rings, and filaments. The shell-like structures seen in the ATCA map still dominate the structure of the neutral atomic ISM in the LMC revealed by the ATCA+Parkes combined map. The previous visual survey of H I shell candidates chosen from the ATCA H I data cube (Kim et al. 1999) has been re-investigated from the current combined data set. Here we confirm that the H I supergiant shells reported in Kim et al. (1999) are indeed seen in the combined data sets and presented in Figure 6 in this paper. Figure 6 is the same as Figure 2 of Kim et al. (1999).

The physical parameters of the individual supergiant shells are summarized in Table 1. The mean column density of neutral hydrogen in the LMC is in the order of $2.8 \times 10^{21}$ $\mathrm{cm}^{-2}$. Assuming a mean column density is distributed uniformly over the disk thickness of 
$\Delta=360$ pc (Kim et al. 1999), we estimate a mean gas particle density of $n_{H} \sim 2 \mathrm{~cm}^{-3}$. We may estimate the amount of kinetic energy of the $\mathrm{H}$ I gas associated with the expanding $\mathrm{H}$ I shells, using their derived sizes and expansion velocities. A total predicted kinetic energy of the interstellar gas associated with $\mathrm{H}_{\mathrm{I}}$ expanding supershells is $3.9 \pm 1.3 \times 10^{53}$ ergs over the mean dynamical age of the expanding shell $\sim 6$ Myr. This result is remarkably similar to the total kinetic energy deposited from the stellar winds $\sim 4.3 \times 10^{53}$ ergs over $6 \mathrm{Myr}$,

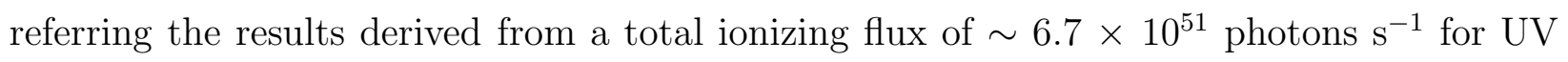
sources in the LMC (Smith et al. 1987) and using the relationship between the ionizing photon flux and the stellar wind mechanical luminosity, $L_{w} / N_{c}=3.2 \times 10^{-13}$ ergs photon $^{-1}$ (Wilson 1983).

The distribution by number of the $\mathrm{H}$ I shells as a function of their radius is presented in Figure 17 of Kim et al. (1999). In the range $100-1000$ pc, the data are consistent with a power-law distribution of slope $s=-1.5 \pm 0.4$. In order to compare the size spectrum of the $\mathrm{HI}$ shells in the LMC with a size spectrum of $\mathrm{H}$ I clouds, we investigate the $\mathrm{HI}$ cloud candidates from the ATCA+Parkes combined data cube. It is well known that the interstellar medium, atomic as well as molecular, is distributed in a hierarchical ensemble of clouds. Such clouds or clumps could be a condensation formed during the thermally unstable cooling. The gas may initially be in either an atomic or a molecular state depending on the local physical conditions (density, excitation temperature, etc.). As the volume densities increase, the gas phase turns to molecular. Recent $\mathrm{CO}(J=1-0)$ and $\mathrm{CO}(J=2-1)$ study of the two nearly face-on galaxies NGC 628 and NGC 3938 shows that the velocity dispersion is remarkably constant with radius, $6 \mathrm{~km} \mathrm{~s}^{-1}$ for NGC 628 and $8.5 \mathrm{~km} \mathrm{~s}^{-1}$ for NGC 3938, and of the same order as the H I velocity dispersion (Combes \& Becquaert 1997). The similarity of the $\mathrm{CO}$ and $\mathrm{H}$ I dispersions suggests that the two components are well mixed, and are only two different phases of the same kinematical gas component. The position of H I clumps can be well matched with CO emission maps (Cohen et al. 1988; Israel et al. 1993; Fukui et al. 1999). A large fraction of the H I clumps have a detectable $100 \mu \mathrm{m}$ emission. Similarly, where the H I clumps are more intense, and the clumps are associated with CO clouds. The large-scale association between $\mathrm{H}$ I and CO is clearly of central interests for studies of cloud and star formation in galaxies (Elmegreen \& Elmegreen 1987).

Shapes of H I clouds are difficult to define and the identification of clouds is still a subjective issue. However, majority of H I clouds can be redefined as H I clumps at small scales in either larger sheets or filaments abound rather than spherical blobs. The present H I aperture synthesis survey of the LMC is particularly well suited to reach the statistical characteristics of H I clouds or clumps. Since the distance of the LMC is known as $55 \mathrm{kpc}$ (Feast 1991) and the LMC is nearly face-on disk galaxy so that confusion along the line of sight is negligible. We have identified and catalogued H I clouds in the LMC by defining 
a cloud to be an object composed of all pixels in right ascension, declination, and velocity that are simply connected and that lie above the threshold brightness temperature (Scoville et al 1987; Lee et al. 1990; Lee et al. 1997). We applied this method rather than gaussian clumping method as the other may generate all gaussian clumps which are not realistic in general. In fact, most of clouds do not have gaussian profiles, and especially it is true for HI clouds. Ideally, one would like to define clouds with a $0 \mathrm{~K}$ threshold temperature. However, low threshold temperatures are impractical in view of the noise level in the spectra and more importantly because of the blending of adjacent clouds which often occurs in crowded regions. We have found $\mathrm{H}$ I clouds or clumps using the automatic clump identification code with three thresholds of the brightness temperature, $T_{B}=16 \mathrm{~K}\left(\approx 5 \times T_{R M S}\right), 32 \mathrm{~K}, 64 \mathrm{~K}$. Final selection of the $\mathrm{H}$ I clouds has been made with high-temperature thresholds in order to reduce the blending of emission from unrelated clouds.

The distribution by number of the H I clouds as a function of their size is presented in Figure 7. The derived sizes of clouds are distributed in a wide range of scales $20-400$ pc. The sizes are computed as the square root of the area. Peak of their size distribution of H I clouds or clumps resides in $20-30$ pc. However, the effective synthesis beam size $1^{\prime}$ limits the size distribution of the smallest H I clouds. The data are consistent with a power-law distribution with slope of between $s=-0.9 \pm 0.2$ and $s=-1.1 \pm 0.2$. Compared to the slope found from the size spectrum of CO clouds in the inner Galaxy, $-2.43 \pm 0.05$ (Solomon et al. 1987); Ophiuchus, -2.20 \pm 0.06 ; Rosette, -2.26 \pm 0.08 ; Maddalena-Thaddeus, $-2.46 \pm 0.20 ; \mathrm{M} 17,-2.09 \pm 0.18$ (Elmegreen \& Falgarone 1996), the power-law distribution of the size spectrum of $\mathrm{H}$ I clouds is flatter than the typical size spectrum of $\mathrm{CO}$ clouds in the Milky Way.

The measured perimeter $P$ and measured enclosed area $A$ of each H I cloud in a log$\log$ plot (Figure 8) gives a set of points lying along with a slope of $D / 2=0.73 \pm 0.1$. The relation between area and perimeter of each identified $\mathrm{H}$ I cloud, $P \propto A^{D / 2}$, can determine the fractal dimension $D=1.47 \pm 0.2$ of a cloud boundary (Vogelaar \& Wakker 1994; Williams, Blitz, \& McKee 2000). The measured fractal dimension of H I clouds in the LMC is a similar dimension, $D \pm 1.4$, found in many studies of the molecular ISM (Falgarone et al. 1991; Williams, Blitz, \& McKee 2000). For clouds identified with different thresholds of the brightness temperature, the fractal dimension $D$ found from the relation between area and perimeter is invariant as shown in Figure 8.

We find that a previous analysis of the holes and shells in the H I distribution shows the same power-law behavior with that of clumps. The implication of the result is that the formation of the filament-like or shell-like structures, as well as clumps could be formed with the same power-law for dependence on the mass of gas in the galaxy and the stellar energy 
feedback. Satisfactory theoretical explanation needs to be developed for this observational fact.

\section{Summary}

We present the merged images of the LMC from the H I Parkes multibeam observations with the aperture synthesis mosaic made by combining data from 1344 separate pointing centers using the Australia Telescope Compact Array (ATCA). The images are constructed by a Fourier-plane technique and sensitive to structure on scales of $15 \mathrm{pc}$ (for an LMC distance of $55 \mathrm{kpc}$ ) upwards. The addition of total power data reveals the plateau of diffuse H I emission. We find that total power data is important in recovering the true source structures even linear mosaicing technique recovers the 'missing' short spacings in the $u v$ plane (Ekers \& Rots 1979; Cornwell 1988). The structure of the neutral atomic ISM in the LMC reveals the clumpiness of the $\mathrm{H}$ I distribution over the whole of the LMC. We have identified and catalogued H I clouds in the LMC by defining a cloud to be an object composed of all pixels in right ascension, declination, and velocity that are simply connected and that lie above the threshold brightness temperature. The power-law distribution of the size spectrum of H I clouds is similar to the typical size spectrum of the holes and shells in the $\mathrm{H}$ I distribution.

We are indebted to the other team members of ATCA HI mosaic project Mike Kesteven, and Dave McConnell. We thank Bruce Elmegreen and Martin White for interesting discussions. We appreciate anonymous referee for improvement of this paper.

\section{REFERENCES}

Alves, D.R., \& Nelson, C.A. 2000, ApJ, 542, 789

Bajaja, E., \& van Albada, G.D. 1979, A\&A, 75, 251

Chu, Y.-H., \& MacLow, M.-M. 1990, ApJ, 365, 510

Cohen, R.S., Dame, T.M., Garay, G., Montani, J., Rubio, M., \& Thaddeus, P. 1988, ApJ, 331,95

Combes, F., \& Becquaert, J.-F. 1997, ApJ, 486, 79

Cornwell, T.J. 1988, A\&A, 202, 316

de Blok, W. J. G., McGaugh, S. S., Bosma, A., \& Rubin, V. C. 2001, AJ, 552, L23 
Deul, E.R., \& den Hartog, R.H. 1990, A\&A, 229, 362

de Vaucouleurs, G., \& Freeman, K.C. 1972, Vistas in Astronomy, 14, 163

Dopita, M.A. 1998, Lecture Note

Dopita, M. A., Mathewson, D. S., \& Ford, V. L. 1985, ApJ, 297, 599

Efremov, Y. N., Ehlerová, S., \& Palous , J. 1999, 350, 457

Ekers, R.D., \& Rots, A.H. 1979, in Imaging Formation from Coherence Functions in Astronomy, eds. van Schooneveld, C. (Dordrecht: Reidel), p.61

Elmegreen, D.M., \& Elmegreen, B.G. 1987, ApJ, 314, 3

Elmegreen, B.G.,\& Falgarone, E. 1996, ApJ, 471, 816

Elmegreen, B.G., Kim, S., \& Staveley-Smith, L. 2001, ApJ, 548, 749

Falgarone, E., Phillips, T.G., \& Walker, C.K. 1991, ApJ, 378, 186

Feast, M.W. 1991, in The Magellanic Clouds:Proceedings of the 148th Symposium of the International Astronomical Union, eds. Haynes, R., \& Milne, D. (Dordrecht: Reidel), p.1

Gardiner, L.T., Turfus, C., \& Putman, M.E. 1998, ApJ, 507, 35

Heiles, C. 1979, ApJ, 229, 533

Heiles, C. 1984, ApJS, 55, 585

Hindman, J.V. 1967, Aust. J. Phys., 20, 147

Holdaway, M.A. 1999, in Mosaicing with Interferometric Arrays, eds. by Taylor, G.B., Carilli, C.L., \& Perley, R.A. (ASP Conference Series), Vol. 180, p.20

Israel, F.P. et al. 1993, A\&A, 276, 25

Kamphuis, J.J., \& Sancici, R. 1993, A\&A, 273, L1

Kim, S., Staveley-Smith, L., Dopita, M.A., Freeman, K.C., Sault, R.J., Kesteven, M.J., \& McConnell, D. 1998, ApJ, 503, 674

Kim, S., Dopita, M.A., Staveley-Smith, L., \& Bessell, M. 1999, AJ, 118, 2797

Lee, Y., Jung, J., \& Kim, H.G. 1997, PKAS, 12, 111

Luks, T., \& Rohlfs, K. 1992, A\&A, 263, 41

McGee, R.X., \& Newton, L.M. 1986, PASA, 6, 471

McGee, R.X., \& Milton, J.A. 1966, Aust. J. Phys., 19, 343

Olling, R. P. 1996, AJ, 112, 481

Olsen, K.A.G., Kim, S., \& Buss, J.F. 2001, AJ, 121, 3075

Padoan, P., Kim, S., Goodman, A., Staveley-Smith, L. 2001, 555, 33

Points, S.D., Chu, Y.-H., Kim, S., Smith, R.C., Snowden, S.L., Brandner, W., Gruendl, R.A. 1999, ApJ, 518, 298

Puche, D., Westpfhal, D., Brinks, E., \& Roy, J. 1992, AJ, 103, 1841

Putman, M. et al. 1998, Nature, 394, 752

Sault, R. J., Staveley-Smith, L., Brouw, W. N. 1996, A\&A, 120, 375

Schwarz, U.J., Wakker, B.P. 1991, in Radio Interferometry: Theory, Techniques and Applications, Cornwell, T.J., Perley, R.A. eds. ASP Conf. Series, San Francisco, p. 188 
Schwering, P.B.W., \& Israel, F.P. 1989, A\&A, 79, 79

Shostak, G.S., \& Skillman, E.D. 1989, A\&A, 214, 33

Smith, A.D., Cornett, R.H., \& Hill, R.S. 1987, 320, 609

Solomon, P. M., Rivolo, A. R., Barrett, J., \& Yahil, A. 1987, ApJ, 319, 730

Stanimirovic, S., Staveley-Smith, L., Dickey, J.M., Sault, J., \& Snowden, S.L. 1999, MNRAS, 302, 417

Staveley-Smith, L., Sault, R.J., Hatzidimitriou, D., Kesteven, M.J., \& McConnell, D. 1997, MNRAS, 289, 225

Staveley-Smith, L., Kim, S., Bruns, C., Calabretta, M.R., Haynes, R.F., Kalnjas, A., Kesteven, M.J., \& Wilson, W.E. 2002, in preparation.

Tenorio-Tagle, G., \& Bodenheimer, P. 1980, ARA\&A, 26, 145

van der Marel, R.P., \& Cioni, M.L. 2000, AJ, 122, 1807

Vogelaar, M.G.R., \& Wakker, B.P. 1994, A\&A, 291, 557

Wada, K., Spaans, M., Kim, S., \& Norman, C. A. 2000, ApJ, 540, 797

Walter, F., \& Brinks, E. 2001, AJ, 121, 3026

Weinberg, M.D. 2000, ApJ, 532, 922

Westerlund, B.E., \& Mathewson, D.S., 1966, MNRAS, 131, 371

Williams, J.P., Blitz, L., \& McKee, C.F. 2000, in Protostars and Planets IV, eds. Mannings, V., Boss, A.P., \& Russell, S.S. (Tucson: University of Arizona Press), p. 97

Wilner, D.J., \& Welch, W.J. 1994, ApJ, 427, 898

Ye, T., \& Turtle, A.J. 1991, MNRAS, 249, 693

Zhao, H.S., \& Evans, N.W. 2000, ApJ, 545, 35

This preprint was prepared with the AAS IATEX macros v5.2.

Fig. 1.- A representation of the feathering process in the Fourier domain. This gives the expected visibility function of a point source from the Parkes and ATCA observations. The feathering process filters out the short spatial frequencies of the ATCA data, so that the sum of this and the Parkes data give a good representation of the object at all spatial frequencies. 
Fig. 2.- The individual channel maps for the H I datacube in the LMC. The heliocentric velocity is marked at the top left in each panel. Each panel is the average of five adjacent channels of width $1.649 \mathrm{~km} \mathrm{~s}^{-1}$ giving a panel spacing of $8.2 \mathrm{~km} \mathrm{~s}^{-1}$. The pots cover most of the $\mathrm{H}$ I emission in the LMC from $210-334 \mathrm{~km} \mathrm{~s}^{-1}$. Black represents regions with the highest brightness temperatures of $136.7 \mathrm{~K}$; white represents $0 \mathrm{~K}$.

Fig. 2.- continued.

Fig. 2.- continued.

Fig. 2.- continued.

Fig. 3.- The peak H I surface brightness map for the LMC. The grey scale intensity range is 0 to $136.7 \mathrm{~K}$. This map is sensitive to the small $\mathrm{H}$ I clouds with the highest opacity along the line of sight. In such regions, the brightness temperature will approach the spin temperature of the H I . The image is similar to the ATCA-only image of Kim et al. (1998) and emphasizes the filamentary, bubbly and flocculent structure of the ISM in the LMC.

Fig. 4. - The H I column density image of the LMC from the combined ATCA and Parkes data. The intensity range is 0 to $7.3 \pm 0.3 \times 10^{21} \mathrm{H}$-atom $\mathrm{cm}^{-2}$ in $\mathrm{H}$ I column density.

Fig. 5.- Declination Velocity images of the LMC. Each panel is a slice through the LMC data cube at $\mathrm{pa}=0^{\circ}$ at the RA specified at the top of each panel. The RA separation of the slices is $\sim 2.0^{\mathrm{m}}$ or $\sim 10$ beamwidths, and the width of each slice is $23^{\mathrm{s}}$, or $\sim 2$ beamwidths. Only a fraction of the data are shown. The grey scale intensity range is 0 to $136.7 \mathrm{~K}$.

Fig. 5.- continued.

Fig. 5.- continued.

Fig. 5.- continued.

Fig. 6.- Supergiant shells are overlayed on the peak brightness HI map.

Fig. 7.- Histograms of H I clump size for three thresholds of the brightness temperature, $T_{B}=16 \mathrm{~K}, 32 \mathrm{~K}, 64 \mathrm{~K}$. 
Fig. 8.- A log-log plot of the measured perimeter versus the measured area in units of pixels of H I clump for three thresholds of the brightness temperature, $T_{B}=16 \mathrm{~K}, 32 \mathrm{~K}, 64 \mathrm{~K}$.

Table 1: List of positions, radii and heliocentric velocities for the H I supergiant shells (SGSs) identified in the LMC.

\begin{tabular}{lccccc}
\hline \hline Shell & RA & DEC & $\begin{array}{c}\text { Shell } \\
\text { Radius } \\
\left({ }^{\prime}\right)\end{array}$ & $\begin{array}{c}\text { Expanding } \\
\text { Velocity } \\
\left(\mathrm{km} \mathrm{s}^{-1}\right)\end{array}$ & $\begin{array}{c}\text { Heliocentric } \\
\text { Velocity } \\
\left(\mathrm{km} \mathrm{s}^{-1}\right)\end{array}$ \\
\hline SGS1 & $04: 58: 36$ & $-73: 33: 57$ & $24.3 \times 22.7$ & 19.0 & 253 \\
SGS2 & $04: 58: 30$ & $-68: 39: 29$ & $18.7 \times 18.7$ & 17.0 & 272 \\
SGS3 & $04: 59: 41$ & $-65: 44: 43$ & $26.9 \times 26.9$ & 15.0 & 294 \\
SGS4 & $05: 02: 51$ & $-70: 33: 15$ & $36.7 \times 28.3$ & 23.0 & 241 \\
SGS5 & $05: 04: 08$ & $-68: 31: 55$ & $36.6 \times 36.6$ & 13.0 & 292 \\
SGS6 & $05: 13: 58$ & $-65: 23: 27$ & $49.0 \times 37.5$ & - & 300 \\
SGS7 & $05: 22: 42$ & $-66: 05: 38$ & $32.7 \times 11.2$ & - & 305 \\
SGS8 & $05: 23: 05$ & $-68: 42: 49$ & $15.0 \times 15.0$ & 22.5 & 271 \\
SGS9 & $05: 25: 46$ & $-71: 09: 16$ & $30.7 \times 30.7$ & - & 235 \\
SGS10 & $05: 30: 45$ & $-68: 04: 30$ & $32.7 \times 26.5$ & - & 284 \\
SGS11 & $05: 31: 33$ & $-66: 40: 28$ & $38.7 \times 37.7$ & 36.0 & 306 \\
SGS12 & $05: 30: 26$ & $-69: 07: 56$ & $41.1 \times 29.0$ & - & 269 \\
SGS13 & $05: 30: 30$ & $-68: 48: 28$ & $14.5 \times 21.2$ & - & \\
SGS14 & $05: 34: 33$ & $-66: 20: 15$ & $15.4 \times 15.4$ & - & 305 \\
SGS15 & $05: 34: 44$ & $-68: 44: 36$ & $17.9 \times 17.9$ & 24.0 & 279 \\
SGS16 & $05: 36: 54$ & $-68: 27: 46$ & $15.3 \times 15.3$ & 18.0 & 269 \\
SGS17 & $05: 40: 26$ & $-68: 18: 58$ & $33.3 \times 26.7$ & - & 292 \\
SGS18 & $05: 41: 00$ & $-71: 15: 18$ & $30.0 \times 26.7$ & - & 223 \\
SGS19 & $05: 41: 27$ & $-69: 22: 23$ & $26.0 \times 26.0$ & - & 259 \\
SGS20 & $05: 46: 49$ & $-70: 02: 32$ & $24.7 \times 24.7$ & - & 259 \\
SGS21 & $05: 44: 53$ & $-66: 28: 29$ & $13.0 \times 13.0$ & 19.0 & 299 \\
SGS22 & $05: 46: 16$ & $-68: 22: 02$ & $14.7 \times 14.7$ & 21.5 & 303 \\
SGS23 & $05: 51: 16$ & $-67: 37: 18$ & $41.3 \times 41.3$ & 23.0 & 296 \\
\hline & & & & & \\
\hline
\end{tabular}


This figure "fig1.gif" is available in "gif" format from: http://arxiv.org/ps/astro-ph/0506224v1 
This figure "fig3.gif" is available in "gif" format from: http://arxiv.org/ps/astro-ph/0506224v1 
This figure "fig6.gif" is available in "gif" format from: http://arxiv.org/ps/astro-ph/0506224v1 
This figure "fig7.gif" is available in "gif" format from: http://arxiv.org/ps/astro-ph/0506224v1 
This figure "fig8.gif" is available in "gif" format from: http://arxiv.org/ps/astro-ph/0506224v1 